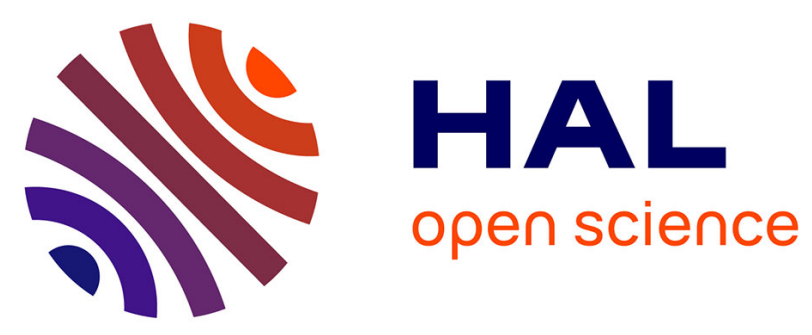

\title{
Translational research in radiobiology in the framework of France HADRON national collaboration
}

\author{
Claire Rodriguez-Lafrasse, Yannick Saintigny, François Chevalier, Myriam \\ Bernaudin, Carine Laurent, Frédéric Pouzoulet, Yolanda Prezado, Frédérique \\ Mégnin-Chanet, Agnès François, Olivier Guipaud, et al.
}

\section{To cite this version:}

Claire Rodriguez-Lafrasse, Yannick Saintigny, François Chevalier, Myriam Bernaudin, Carine Laurent, et al.. Translational research in radiobiology in the framework of France HADRON national collaboration. Translational Cancer Research, 2017, 6 (S5), pp.S795-S806. 10.21037/tcr.2017.06.33 . hal-01610093

\section{HAL Id: hal-01610093 https://hal.science/hal-01610093}

Submitted on 4 Oct 2017

HAL is a multi-disciplinary open access archive for the deposit and dissemination of scientific research documents, whether they are published or not. The documents may come from teaching and research institutions in France or abroad, or from public or private research centers.
L'archive ouverte pluridisciplinaire HAL, est destinée au dépôt et à la diffusion de documents scientifiques de niveau recherche, publiés ou non, émanant des établissements d'enseignement et de recherche français ou étrangers, des laboratoires publics ou privés. 


\title{
Translational research in radiobiology in the framework of France HADRON national collaboration
}

\author{
Claire Rodriguez-Lafrasse ${ }^{1,2}$, Yannick Saintigny ${ }^{3}$, François Chevalier ${ }^{3}$, Myriam Bernaudin $^{4}$, Carine \\ Laurent $^{5,6}$, Frédéric Pouzoulet ${ }^{7,8}$, Yolanda Prezado ${ }^{9}$, Frédérique Megnin-Chanet ${ }^{8,10}$, Agnès François ${ }^{11}$, \\ Olivier Guipaud $^{11}$, Michael Beuve ${ }^{12}$, Jacques Balosso ${ }^{13,14}$
}

${ }^{1}$ Univ Lyon, Université Lyon 1, UMR CNRS 5822 /IN2P3, IPNL, PRISME, Laboratoire de Radiobiologie Cellulaire et Moléculaire, Faculté de Médecine Lyon-Sud, F-69921 Oullins cedex, France; ${ }^{2}$ Hospices Civils de Lyon, Centre Hospitalier Lyon-Sud, 69495 Pierre-Bénite, France; ${ }^{3}$ Commissariat à l'Energie Atomique et aux Energies Alternatives, UMR 6252, CIMAP, LARIA, F-14076 Caen, France; ${ }^{4}$ Normandie Univ, UNICAEN, CEA, CNRS, ISTCT/CERVOxy group, 14000 Caen, France; ${ }^{5}$ SAPHYN (ARCHADE Program), 14076 Caen, France; ${ }^{6}$ Normandie Univ, UNICAEN, UNIROUEN, ABTE, 14000 Caen, France; ${ }^{7}$ Institut Curie, PSL Research University, Translational Research Department, Experimental Radiotherapy Platform, 91405 Orsay, France; ${ }^{8}$ Paris Sud University, Paris-Saclay University, 91405 Orsay, France; ${ }^{9}$ IMNC-UMR8165/CNRS-Paris 11 -Paris 7/NARA team, Campus d'Orsay, 91405, Orsay, France; ${ }^{10}$ INSERM U1196/CNRS UMR9187, bât 112, Centre Universitaire, 91405 Orsay, France; ${ }^{11}$ Institute for Radiological Protection and Nuclear Safety (IRSN), PRP-HOM, SRBE, L3R, 92260 Fontenay-aux-Roses, France; ${ }^{12}$ Univ Lyon, Université Lyon 1, UMR CNRS 5822/IN2P3, IPNL, PRISME, PHABIO, 69322 Villeurbanne, France; ${ }^{13}$ Université de Grenoble Alpes, F-38400 Saint Martin d'Hères, Grenoble, France; ${ }^{14}$ France HADRON, IPNL Centre Universitaire de la Doua, F-69622 Villeurbanne, France

Contributions: (I) Conception and design: All authors; (II) Administrative support: All authors; (III) Provision of study materials or patients: All authors; (IV) Collection and assembly of data: All authors; (V) Data analysis and interpretation: All authors; (VI) Manuscript writing: All authors; (VII) Final approval of manuscript: All authors.

Correspondence to: Prof. Claire Rodriguez-Lafrasse. Laboratoire de Radiobiologie Cellulaire et Moléculaire, CNRS/IN2P3, IPNL, Faculté de Médecine-Lyon-Sud BP12, 69921 OULLINS Cedex, France. Email: claire.rodriguez-lafrasse@univ-lyon1.fr.

\begin{abstract}
France HADRON is a French national collaboration that brings together 26 teams in nine different French cities (Lyon, Caen, Clermont-Ferrand, Paris-Orsay, Nice, Toulouse, Marseille, Strasbourg and Grenoble). This infrastructure was created in 2013 and follows the ETOILE and ARCHADE projects; it is presently funded by public money. Ten teams, from different scientific background, using a wide range of approaches, participate in radiobiology researches upon protons and carbon ion therapy in comparison with photons used in classical radiation oncology. These approaches combine the use of multiple biological models of radioresistant tumors and healthy tissue critical for treatment tolerance. Thus, the determinants of the radioresistance of head and neck squamous cell carcinoma (HNSSC), high-grade gliomas, sarcomas including chondrosarcomas and the ubiquitous endothelial cells and fibroblasts are studied. Multiple methodological approaches are implemented. In particular, these include: the extraction of tumor stem cell populations and their radiobiological comparison with the differentiated tumor cells; the studies in hypoxic and normoxic conditions; the development and the use of models of 3D cell cultures that highlight important differences in cell behaviors; the cell biology with the analysis of typical neoplastic mechanisms such as invasive and cell migration processes, as well as late recovery of irradiated tumor cells that reenter proliferation; the molecular biochemistry with analysis of resistance signaling pathways, telomerase regulation and genomic analysis of predictors of radioresistance in retrospective and prospective series of patients (gliomas, HNSSC, sarcomas) and finally the oxidative stress, genotoxicity and inflammatory measurements in normal human cells at early and late times after irradiation. This work allows a better understanding of the mechanisms of cellular and tumor radioresistance, normal tissue toxicity, but they also enable pre-clinical and clinical approaches. Thus, experimental approaches of combinations of pharmacological treatments acting on the identified resistance or toxicity mechanisms with the different types of radiation studied are developed. The comparison between stem cells and differentiated cells is very interesting in this respect. This information, combined with phenomenological data on survival and relative biological effectiveness (RBE), contributes to the adjustment and parameterization of bio-mathematical predictive models of the cellular response such as the $\operatorname{Nanox}^{\circledR}$
\end{abstract}


model. This work enables, on the one hand, to suggest hypotheses of clinical trials aimed at acting on tumor radioresistance; and, on the other hand, to integrate into studies associated with prospective transnational clinical research protocols, comparing carbontherapy $v$ s. photon or proton radiotherapy, in collaboration with the European carbontherapy centers, in particular the Heidelberg Ion Therapy (HIT) in Germany and Centro Nazionale di Adroterapia Oncologica (CNAO) in Italy. The potential of France HADRON in radiobiology is important and can make a useful contribution to this field of research, in particular by pursuing a convergence approach of the methods, the models and the topics investigated.

Keywords: Hadrontherapy; carbon ions; protons; radiobiology; modelization

Submitted Jan 19, 2017. Accepted for publication Jun 09, 2017.

doi: $10.21037 /$ tcr.2017.06.33

View this article at: http://dx.doi.org/10.21037/tcr.2017.06.33

\section{Introduction}

France HADRON is the collaboration of all the scientific teams involved in applied research regarding medical application of particle therapy in France (www.francehadron.fr). It has been progressively built up since the early 2000, first by gathering scientists of Lyon and the Region of Rhône-Alpes in the frame of the ETOILE project (1); then, since 2013, on a full national scale in the frame of France HADRON headed by Prof. Jacques Balosso and integrating the ARCHADE center in Caen (www.archade. fr). Today, it gathers about 26 teams from ten different towns structured as a network with five main nodes jointed to the initial place of Rhône-Alpes: Caen, Nice, Toulouse and Orsay. France HADRON is presently supported by public money in the frame of the so called "Investment for the future". The scientific project is organized in four working packages (Figure 1) namely: clinical research, basic data for modelization, instrumentation and radiobiology (www.france-hadron.fr). This review will only focus on the working package Radiobiology headed by $\mathrm{Pr}$ Claire Rodriguez-Lafrasse and which gathers about ten French teams of research.

\section{Scientific aims, models of study and irradiation facilities}

The radiobiology topics are developed in different laboratories having historically very different domains. A continuous effort is made to organize and set up comparable and complementary works with the following aims (2):

* To elucidate and quantify the molecular events triggered by protons and carbon ions in both tumor and healthy tissues using different models (cellular, pre-clinical and patients) and different time scales (from femtosecond to years);

* To predict the response of tumor and healthy tissues to both types of radiation by the selection and validation of biological markers of radiosensitivity;

* To optimize treatment delivery and evaluate the sensitizing effect of combination with chemotherapy or targeted therapies;

* To improve existing or develop new predictive models to be used for treatment planning and to enrich them with biological parameters.

A critical point for this research program is the access to particle beam of medical quality. France HADRON has devoted a part of its funding to facilitate beam accesses. For protons, two complementary accesses are in construction mainly funded by France HADRON in Nice and Orsay. Meanwhile experiments have to be done on the medical beam lines but also, through the RadioGraff platform, with the $3.5 \mathrm{MeV}$ protons of the Van de Graaff accelerator at Institut de Physique Nucléaire de Lyon (IPNL) $(3,4)$. Concerning experiments with carbon ions, the main source is the Grand Accélérateur National d'Ions Lourds (GANIL) nuclear physics center in Caen, but also the Gesellschaft für Schwerionenforschung (GSI) and the Heidelberg Ion-Beam Therapy Center (HIT) in Germany or more recently the National Institute of Radiological Sciences (NIRS) in Japan. As a whole the beam access remains scarce and slows down the pace of research.

To make possible inter-experiment and inter-team comparisons, an advanced dosimetry has been developed at GANIL to warrant high quality experiments. This methodology has been described in detail by Durantel et al. (5).

Different cellular models established from human tumors, 

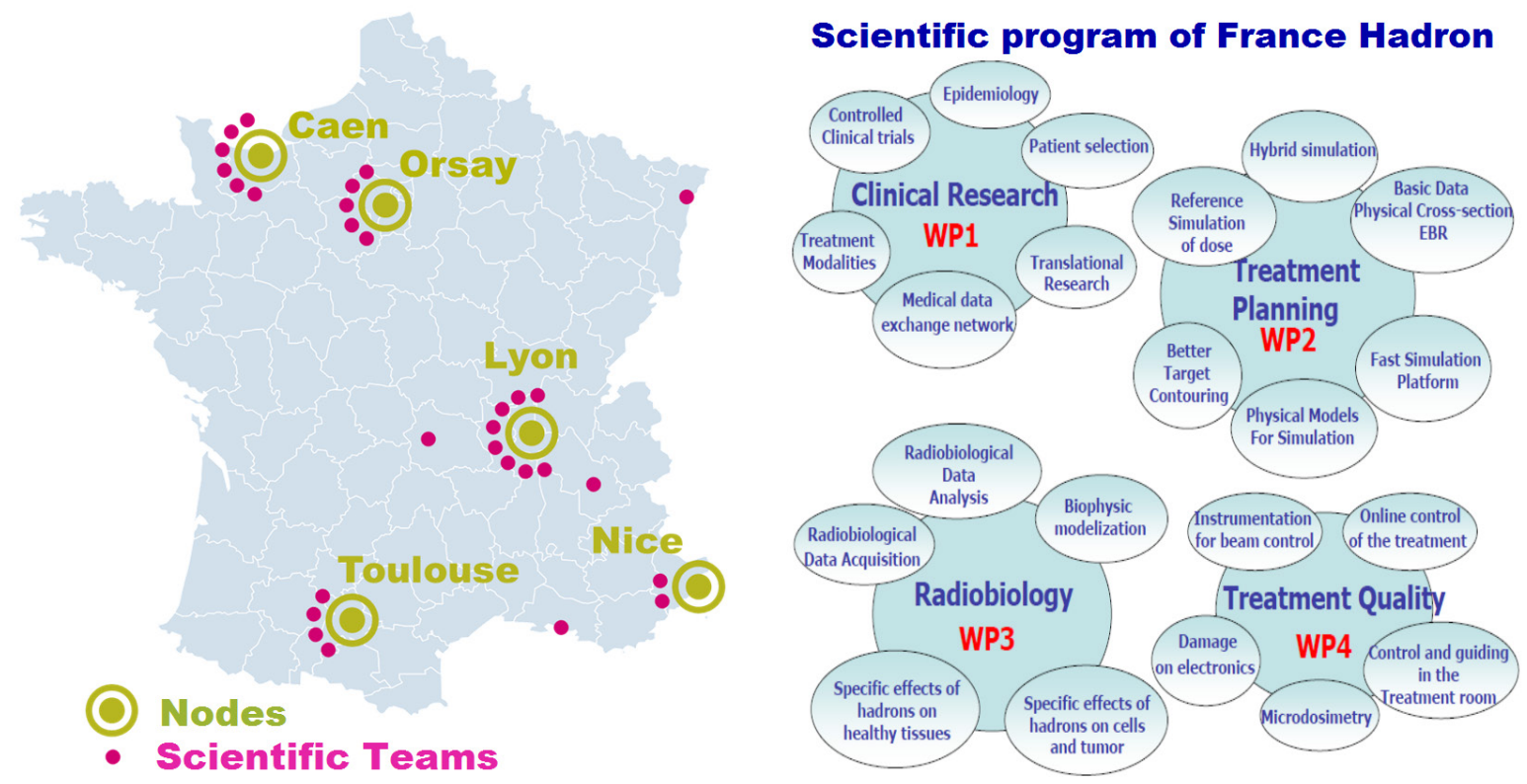

Figure 1 Five main nodes of France HADRON and overview of the four work packages.

which are elective indications of hadrontherapy, normal tissue, and animal models have been used so far. As much as possible clinically related models are chosen mostly in the resistant side of the radiosensitivity range. For head and neck tumor a lot of work has been done with the SQ20B human cell line (6-10) and systematic characterization of multiple cell lines of the same type of tumor have been carried out as for human glioma (11). The cellular response in hypoxic and "physioxic" conditions are explored using either tumor models (12) as spheroids (personal communication) or healthy tissues as chondrocytes (10). The response of the healthy vascular system as well as skin cells is investigated in vitro by molecular studies of human primary endothelial cells and dermal fibroblasts (13). Shared models and methods are future directions to accelerate the production of database and to increase the robustness of the results.

\section{Elucidation and quantification of the molecular events triggered by protons and carbon ions in both tumor and healthy tissues using different models (cellular, pre-clinical and patients) and different time scales (from femtosecond to years)}

\section{Head and neck squamous cell carcinoma (HNSSC) model}

The study of radioresistance has been the first approach of the LRCM group of Lyon in translational radiobiology regarding the research of clues for hadrontherapy. Historically the work began with HNSSC cell lines (7). The tumors of patients with HNSCC have a behavior difficult to apprehend. Despite a relatively good radiosensitivity, the locally advanced cases have a poor local control (35\% at 5 years) with loco-regional and distance relapses, and noteworthy, a relatively disappointing effect of high linear energy transfer (LET) radiations comparatively to other resistant tumors of head and neck such as adenocarcinoma or adenoid cystic carcinoma. This unusual situation obviously urges to deepen understanding of radioresistance of HNSSC (14). The first studies focused on the ceramide-dependent apoptotic pathway. It has been demonstrated that in response to carbon ion irradiation, an early ceramide production is associated with a radiosensitive phenotype leading to apoptosis whereas a late production is associated with radioresistance, mitotic catastrophe and late apoptosis. Ceramide is then a determining factor in the onset of early and late apoptosis after high-LET irradiation and is the mediator of the $\mathrm{p} 53$ independent-apoptotic pathway. Attempting to modulate this pathway could then be an interesting way to improved carbon ion therapy (9).

Another explanation of the worse response and locoregional recurrence of HNSCC tumors could be the presence of the so-called cancer stem cells (CSCs). In order 
to obtain more insight into the mechanisms of tumor escape, the role of CSCs obtained from the SQ20B radioresistant HNSCC cell line (p53 mutated, and HPV negative) was investigated $(10,15,16)$. The hypothesis was that CSCs could be responsible of the radioresistance and the delayed relapse of such tumors (10). A fairly reproducible separation of CSCs from HNSCC cell lines $(16,17)$ allowed to study the complex relationships between radioresistance, tumor relapse and cell migration. It was shown that the resistance of SQ20B-CSCs may result from an imbalance between exacerbated self-renewal and proliferative capacities and the decrease in apoptotic cell death triggering. Attempts to modulate these processes seem therefore to be promising therapeutic strategies (10).

Beyond the possibility of CSC to promote local relapse, another explanation for CSC resistance may be their high migratory and invasive abilities (18). The invasionmigration mechanisms involved in the SQ20B cell line and its CSC subpopulation were compared in response to photonic and hadronic irradiations. For the total SQ20B population, after 2 Gy photon irradiation migration as well as invasion increased by $45 \%$ and $200 \%$ respectively. Carbon ions, independently of the dose, significantly decrease the migration and invasion of SQ20B cells. Regarding the SQ20B-CSC cells, they have a very high migratory and invasive power in basal conditions. Photon irradiation increases their migration and invasiveness at a lower level than parental cells, while irradiation with carbon ions reduces invasion and migration at a level close to significance compared to non-irradiated cells (19).

In the frame of a Lyon-Caen close collaboration, hypoxic and normoxic conditions have been introduced since CSCs are mainly located in hypoxic niches of the tumor. The mechanisms involved in radioresistance and invasion-migration processes were investigated in SQ20B cells but also in FaDu, another HNSCC cell line, as well as their subpopulation of CSCs after exposure to photons or carbon ions. The protein hypoxia-inducible factor $1 \alpha(\mathrm{HIF}-1 \alpha)$ is considered as the major transcriptional regulator of the cellular and developmental response to oxygen homeostasis. In hypoxic conditions, HIF-1 $\alpha$ plays a central role in radioresistance [oxygen enhancement ratio (OER >1.2)] and the increased invasion and migration phenomenon activated by a photonic irradiation. Conversely, since carbon ions appear unable to stabilize $\mathrm{HIF}-1 \alpha$ in CSCs, there is neither resistance linked to the oxygen effect $(\mathrm{OER}=1)$ nor activation of the migration and invasion pathways (20).

\section{Cerebral tumors and bealthy cerebral tissue models}

These tumors are considered as very radioresistant. Some old works about neutrons (21) and the ongoing CINDERELLA clinical trial at HIT are encouraging to probe the effect of carbon ions on such tumors.

The CERVOxy team (a team from the ISTCT unit) at Caen is studying, in conditions of normoxia or hypoxia, the response of glioblastoma (GB) and healthy brain tissue cells exposed to carbon ions (personal communication). GB human cell lines were GL15, U251 and T98G; the murine were C6 and 9L; concerning the cells of the healthy cerebral tissue, these were astrocytes (glial cells). The preliminary results show different radiosensitivity to carbon ions according to the GB cell lines especially in hypoxia and distinct from those in $\mathrm{X}$-rays, but with a higher efficiency of the carbon ions. The latter have an efficacy less affected by hypoxia. Astrocytes (healthy cells) have a high resistance to carbon ions equivalent to that observed in response to X-rays. However, it is of importance to note that rat astrocytes have been used and that further experiments with human healthy astrocytes would be needed since different mechanisms may occurs notably in terms of DNA repair. In addition, the year 2015 was marked by our first in vivo X-ray experiments of rats and mice orthotopically implanted with human GB xenografts.

On parallel experiments, at Villejuif then at Orsay, the systematic comparison of radiosensitivity performed on four GB cell models grown in classical 2D cell conditions or in $3 \mathrm{D}$ spheroïds shows that carbon ions are more efficient to kill GB spheroïds than protons (personal communication).

Also interesting are the effects of irradiation on inflammatory cells infiltrating the tumor where they can represent up to $40 \%$ of the cells of the tumor mass. For the GB, macrophages represent the bulk of inflammatory cells. It has recently been shown that irradiation promotes inflammation and may change the oxygenation level of tumors. CERVOxy has also demonstrated that hypoxia modifies the macrophage phenotype by favoring the M2 pro-tumor phenotype (22) and will study whether X-ray and carbon ion irradiation favors pro-tumor inflammation.

Other works refining knowledge about radioresistance of ocular melanomas have also been carried out (23). This is an important point since this type of tumor has been the most frequently treated yet in France by proton therapy. 


\section{Chondrosarcoma and chondrocyte models}

Another group of Caen, LARIA (a team from CIMAP), carried out the study of the radioresistance of the cells of certain critical tissues, in particular of those having physiologically very low oxygenation (physioxia) (24). Thus, studies have been carried out on chondrosarcoma and healthy cartilage cells in the form of an in vitro 3D model developed in a collagen scaffold (12). Cellular viability and carbon ion radiosensitivity tests (clonogenicity, Western blot, cytometry, oxidative stress) made it possible to characterize the toxicity of carbon ions on healthy cartilage as well as the radiobiological response of cells from different chondrosarcoma lines. Carbon ion irradiation does not induce more senescence on such healthy tissue than irradiation with $\mathrm{X}$-rays (25). In this study, biological effects measured in 2D cultures, such as the higher effectiveness of carbon ions compared to $\mathrm{X}$-rays, seems over-exaggerated compared to the $3 \mathrm{D}$ model and the reality of clinics, especially concerning healthy tissues and primary cell analysis. Thus, this study is the first report investigating the differential impact of carbon ions versus $\mathrm{X}$-rays in human articular cartilage using a $3 \mathrm{D}$ cartilage model which could be useful to assess the RBE of therapeutic beams in healthy tissues and tumors. A study of bystander effects (also called indirect effects) was initiated using irradiated chondrosarcoma cells as signal emitters and immortalized chondrocytes, as signal receptors.

\section{Healthy vascular endothelium model}

During radiotherapy, the vascular endothelium is irradiated in both healthy tissues and the vascularized tumor. Activated by irradiation, endothelial cells are involved in inflammatory reactions induced by tissue damage. In organs at risk, radiation-induced endothelial chronic activation/ dysfunction or loss of integrity may compromise the function of the entire structure. In the tumor, dysfunction and loss of integrity can cause the death of tumor cells and provide a therapeutic benefit. The endothelium is thus considered as a key cell compartment for the response to ionizing radiation of healthy tissue and tumors, and as a promising target to improve the differential effect of radiation therapy in the future. The IRSN's L3R team at Fontenay Aux Roses has long been interested in the role of endothelium in the initiation and the development of tissue damages induced by high doses of gamma or $\mathrm{X}$-rays radiations in the context of radiotherapy. Besides, carbontherapy has attracted attention because of its highly lethal effects. However, the effects of heavy ion irradiation on vascular endothelial cells have been little reported, in particular with regard to molecular pathways involved in endothelial functions and dysfunctions. The question is whether carbon ions impact vascular function more than conventional treatment and, in this case, if it causes a risk of damage to healthy tissue or a benefit for tumor control. In this work, the transcriptional profile of primary human vascular endothelial cells (HUVECs) exposed to high doses of carbon ions has been studied in comparison to ${ }^{137} \mathrm{Cs}$ and $\mathrm{X}$-rays irradiation. HUVECs at confluence were exposed to increasing doses of carbon ions and collected at different times post-irradiation from day 4 to 21. Interestingly, in spite of the RBE of carbon ions $(2.5$ to 3 according to clonogenic cell survival assay), 5-10\% of the exposed cells were still present and metabolically active 3 weeks after exposure. A panel of 192 genes was used to compare molecular profiles using inference and unsupervised multivariate statistical tools such as principal component analysis and hierarchical clustering. This panel was previously established on the basis of the differential expression results from transcriptomic and proteomic time course analyses of HUVECs irradiated with ${ }^{137} \mathrm{Cs}[(26)$ and unpublished results]. Results show that gene signatures among 192 genes are markedly different between ${ }^{137} \mathrm{Cs} /$ $\mathrm{X}$-rays and carbon ions for equivalent doses of radiation. Untangling the molecular pathways is now underway to give insight into the cause of such differences of responses between the two types of radiation.

\section{Healthy skin buman primary fibroblast model}

Skin being the first organ exposed to irradiation, some studies reported skin toxicity after carbon ion therapy. In particular, Yanagi et al. [2010] (27) reported severe acute and late skin complications on patients treated for unresectable bone and soft tissue sarcoma by a carbon ion dose escalation protocol. Oxidative stress is considered as an important pathway in the appearance of late skin reactions. SAPHYN/ABTE-ToxEMAC (Caen) evaluated oxidative stress in normal human skin fibroblasts after carbon ion $v s$. X-ray irradiation. The $\mathrm{RBE}$ of carbon ions $v$ s. $\mathrm{X}$-rays was 4.8 at $\mathrm{D}_{0}$. Surviving fraction at 2 Gy was $71.8 \%$ and $7.6 \%$ for $X$-rays and carbon ions, respectively. Compared with X-rays, immediate DNA damage was less increased after carbon ions, but a late increase was observed at $\mathrm{D}_{10}$. LPO 
products and protein carbonyls were only increased 24 hours after carbon ions. After X-rays, superoxide dismutase activity was strongly increased immediately and on day 14 at $\mathrm{D}_{0 \%}$, catalase activity was unchanged and glutathione peroxidase activity was increased only on day 14. These activities were decreased after carbon ions compared with X-rays. GSH/GSSG was unchanged after $\mathrm{X}$-rays but was decreased immediately after carbon ion irradiation before an increase from day 7. Secretion of IL-6 was increased at late times after X-ray irradiation. After carbon ion irradiation, IL- 6 concentration was increased on day 7 but was lower compared with $\mathrm{X}$-rays at later times. Carbon ion effects on normal human skin fibroblasts seemed to be harmful in comparison with $\mathrm{X}$-rays as they produce late DNA damage, LPO products and protein carbonyls, and as they decrease antioxidant defenses. Mechanisms leading to this discrepancy between the two types of radiation were then investigated, as well as genotoxicity. In particular, transcription studies were performed showing a decrease in antioxidant, inflammatory, apoptosis, cell cycle arrest and senescence gene transcription after carbon-ion compared to X-rays (unpublished results). Moreover, the measurement of excreted 8-oxodG was performed and give information on oxidative waves occurring after carbon ion irradiation as extracellular 8-oxodG is considered as mainly coming from the cytoplasmic nucleotide pool which is an important target of oxidative stress.

\section{Prediction of the response of tumor and healthy tissues to both types of radiation by the selection and validation of biological markers of radiosensitivity}

\section{Biomarkers of response to carbontherapy}

Since carbon ion hadron therapy is restricted to radioresistant patients, photon irradiation resistance biomarkers are needed. Long telomeres and high telomerase activity have been widely associated with photon radioresistance in different types of cancers. The LRCM team of Lyon thus decided to evaluate an enlarged telomeric status as a potential radioresistance biomarker in vitro using cellular models and ex vivo using patient tumor biopsies. In addition, nothing was known about the role of telomeres in carbon ion response. Thus, the telomeric status after both types of irradiation was evaluated. It was shown telomeres play different roles in the cell responses to carbon ion and photon irradiation because of the biophysical properties of each type of irradiation. While the molecular determinant of telomere length's involvement in photon response remains to be elucidated, telomeric status (POT1 index, Telomere Length) seems to be a valuable predictive marker of photon response. Finally, it has been shown that activation of telomerase is an intrinsic factor of radioresistance in photon therapy but not in relation to carbon ions (28).

In view of these results, a new personalized treatment plan was proposed for glioblastoma patients, based on telomere profiling, whereby poor responders with long telomeres should be treated by carbon ion therapy (28).

In the case of chondrosarcoma, biomarkers of response to carbon ion irradiation can be found in the extracellular environment, as stress factors secreted by irradiated cells. These factors, mainly implicated in the propagation of the bystander signal are studied using proteomic tools, as proposed recently (29).

\section{Radiobiology associated with clinical research protocol}

To advocate the introduction of hadrontherapy in human cancer treatment it is necessary to gather arguments and clues about radioresistant conditions. Such a preliminary work which is somehow based on the clinical radiobiological description of tumor behavior is a part of France HADRON medical project. Such reviews have been carried out for all the families of tumor and one of the most recent has been done about pediatric brain tumors (30).

The experience gained in preclinical studies has made possible to associate the first transnational prospective randomized protocol [comparing carbontherapy in collaboration with the CNAO (31) with treatments without carbon-therapy (PHRC-ETOILE)] with the study of biological parameters of tumor radiosensitivity. The objective is to identify predictive markers that may lead to a priori orientation of patients towards carbontherapy. The patients included in this study will constitute a prospective cohort of 250 patients with sarcomas or chordomas (all localizations except chondrosarcomas and chordomas of the base of the skull) or cystic adenoid carcinomas of the head and neck, all non-metastatic, inoperable or in $\mathrm{R} 2$ resection. A secondary objective of the PHRC-ETOILE is thus to constitute a collection of biopsy samples in order to evaluate the interest of new markers (protein or nucleic acids).

Initially, immunohistochemistry protocols for eight types of protein biomarkers were developed and tested on 
a mouse-grafted tumor model and included in paraffin. In addition, different profiles of expression of mRNA by PCR technique were also identified. Protein markers common to the two most commonly selected tumor types are antiapoptotic proteins such as bcl-2/bcl-XL, HSP27 heat shock protein, survivin, or involved in the activation of an anti-apoptotic pathway such as EGFR. Their level of expression is generally correlated with a poor prognosis. From a fundamental point of view, these markers, and more particularly bcl-2, are implicated in the mechanisms of resistance to radio- and/or chemotherapy treatments. Tumor suppressor proteins such as $\mathrm{p} 53$, proliferation markers such as Ki67 and angiogenesis markers such as cyclooxygenase (COX2), have also overexpression correlated with a low 5-year survival rate.

Among these protein markers, overexpression of bcl-2 or $\mathrm{p} 53$ has the characteristic of being representative of the status of radioresistance to photons (conventional radiotherapy), but not to carbon ions. Indeed, several studies have demonstrated that the tumor response to radiation with high LET is independent of the p53 status and the expression level of the bcl-2 protein. These two markers would therefore be more specifically markers of radioresistance to conventional radiotherapy and discriminating for the orientation towards the carbontherapy.

Regarding the study of nucleic markers, Nanostring ${ }^{\circledR}$ technology has been developed for about 10 years in molecular diagnostics. It allows the detection and the quantification of targets (DNA and/or RNA, even proteins) in biological samples and tends to impose itself in oncology for the study of the gene expression by its simplicity, robustness, versatility and analytical performance (reproducibility and accuracy in particular). "Pan Cancer Pathways" and "Pan Cancer Progression" panels will quantify the expression profile of 730 and 740 genes, respectively, involved in signaling pathways and tumor progression from paraffin-preserved tumor samples.

\section{Optimization of treatment delivery and evaluation of the sensitizing effect of combining carbon ions with chemotherapy or targeted therapies}

\section{Optimization of treatment delivery}

\section{Dose rates}

Beyond the advanced concepts of CSCs exposed previously, more simple technical approaches deserve consideration regarding the fairly diverse methods of irradiation presently available. Noteworthy the variations of dose rates have not the same consequences according to the LET of the beams. High LET beam being rather insensitive to dose rate effect on the contrary of low LET radiations (32). This is an important consideration since, for active scanning systems, carbon ions are delivered in less than $1 \mathrm{~s}$ for the deepest parts of the tumor whereas the shallowest parts are irradiated in a few minutes.

\section{Proton minibeam radiation therapy}

The team New Approaches in RAdiotherapy (NARA) proposed an innovation (33), called proton minibeam radiation therapy ( $\mathrm{pMBRT}$ ), which combines the prominent advantages of protons for RT and the remarkable tissue preservation provided by the use of submillimetric field sizes and a spatial fractionation of the dose, as in minibeam radiation therapy (MBRT). Due to proton lateral scattering, each proton minibeam of the array ("tooth of the comb") gets wider as a function of the depth, overlapping at the tumor position. As a consequence, a homogeneous dose distribution can be generated inside the tumor (like in conventional RT), while the concept of spatial fractionation (peaks and valleys) and its advantages are maintained in the irradiated regions of normal tissue. The technical implementation (34) was performed at Curie Institut-Orsay proton therapy center (ICPO). A complete set of dosimetric data in such small proton field sizes to guide the first biological studies was obtained. In a first experiment, the brain of young adult rats was irradiated with clinical relevant energies. One group of animals received conventional seamless proton irradiation (25 Gy in one fraction). The other group was irradiated with pMBRT (58 Gy peak dose in 1 fraction). A magnetic resonance imaging (MRI) followup as well as histological analysis were performed. Rats treated with conventional proton irradiation exhibited severe moist desquamation, permanent epilation and important brain damage. In contrast, the pMBRT group presented no skin damage, a reversible epilation and no significant brain damage was observed after a 7-month follow up (35). In addition, preliminary results of irradiations of RG2 glioma bearing rats showed a significant increase of lifespan. These first results suggest that the net gain in normal tissue of pMBRT can foster one of the main applications of proton therapy, paediatric oncology, as well as open the door to an efficient treatment 
of very radioresistant tumors, which are currently mostly treated palliatively. Next steps will be to perform more complete evaluations on tumor control as well as studies to unravel the biological mechanisms involved in the remarkable normal tissue sparing.

\section{Combination of carbon ions with chemotherapy or targeted therapies}

Knowledge regarding radioresistance pathways is naturally a source of investigations to develop countermeasures to overcome radioresistance. Such approaches are thus developed in different ways.

A very large part of the radioresistance is occurred by the passive effect of radical scavengers in the cells. Although any variation of the level of this biochemical radioprotection is very touchy and would be extremely toxic for normal cells, it is interesting to study the effect produced by the depletion of glutathione in HNSCC cell lines. A strong sensitization is obtained and, consistently enough, also a reduction of the mutation rate that is wiped out by the increased of cell death (8).

Another way would be to specifically target the CSCs. Concerning the exacerbated self-renewal pathway, a fundamental role of Bmi-1 was demonstrated in SQ20BCSCs for which its inhibition significantly enhanced sensitivity to high and low LET radiation by triggering apoptotic cell death. It was demonstrated that the radioresistance of SQ20B-CSCS could result from their high proliferative capacity related to high aldehyde dehydrogenase (ALDH) activity. The inhibition of ALDH using all-trans retinoic acid (ATRA) induced differentiation of CSCs associated with a significant decrease in cell survival after either carbon or photon irradiation. Regarding apoptosis, since irradiated-CSCs do not undergo early apoptosis because of a transient arrest in G2/M followed by mitotic catastrophe, treatment of CSCs with the checkpoint kinase (Chk1) inhibitor UCN-01 triggered early apoptosis thus leading to radiosensitization after photon and carbon ion exposure. Finally, the combination of ATRA and $\mathrm{UCN}-01$ treatment with carbon ion irradiation drastically decreased the survival fraction at 2 Gy (SF2) of SQ20BCSCs (10).

Moreover, since targeted therapies now take a major place in anti-cancer treatment, particularly in HNSCC, the association of radiation with cetuximab, a mouse human chimeric monoclonal antibody directed against EGF-R was studied. Cetuximab associated with photon or with carbon ions irradiation radiosensitized SQ20B cells. At the opposite, no radiosensitization was observed in CSCs since EGF-R, the target of cetuximab, is underexpressed in the CSC population. The cell invasionmigration tests were carried out and, for the SQ20B cells, after photon irradiation in the presence of cetuximab, migration as well as invasion decreased when compared to photon irradiation alone. Moreover, the significant decrease of migration and invasion observed in response to carbon ions was strongly enhanced by cetuximab. For the SQ20B-CSC, the addition of cetuximab before irradiation with carbon ions did not alter migration, but reduced invasion compared to hadronic irradiation alone. As a whole, the SQ20B-CSC cells resist photonic irradiation and cetuximab and possess a much greater invasiveness than the SQ20B parental cells. The association of carbon ions and cetuximab appears to be effective in decreasing invasion (19).

In conclusion, whatever the pharmacological strategies used, an important radiosensitization of HNSCC-CSCs was obtained. Moreover such a possibility is also studied and discussed for malignant glioma for instance (36). Adjuvant treatments targeting either the inhibition of survival/selfrenewal pathways or the triggering of apoptosis should improve the results for patients treated with photon- or hadrontherapy.

\section{Combining metallic nanoparticles and hadrontherapy}

Actually, since some times already, studies have been suggested and carried out with protons or heavy ions as irradiation and heavy atoms, such as platinum, as radiosensitizers (37). The continuation of such works with nanoparticles is on the way and will certainly take some more places in the future in France HADRON, in particular to enhance proton efficiency on radioresistant tumors. The first attempts are presently in development either outside of France HADRON (38) or inside (39).

\section{Improving or developing predictive models to be used for treatment planning and enriching them with biological parameters}

\section{RBE measurements}

At IGR and Curie Institute (40), a systematic study of $\mathrm{RBE}$ is still going on. In vitro experiments using the 
$76 \mathrm{MeV}$ proton beam line of the ICPO and carbon ion beam at 75 or $95 \mathrm{MeV} / \mathrm{n}$ at GANIL are carried out. The aim of these studies is to compare in correlation with RBE, the influence of culture geometry (2D and 3D spheroids), DNA repair, the expression of post-irradiation proteins in four glioblastoma cell lines after proton and carbon irradiation relative to photons.

Several other RBE measurements are carried out for constraining models and calculation algorithms by different groups in Paris, Caen and Lyon.

\section{Large scale biological data acquisition to improve predictive models}

A large and systematic study of eleven human GB cell lines with gradual radiosensitivity has been carried out to improve models of prediction. The photon irradiation was compared to a $75 \mathrm{MeV} / \mathrm{n}$ carbon ion irradiation at GANIL. The SF2, the radiation dose required to obtain $10 \%$ survival $\left(\mathrm{D}_{10}\right)$, and the carbon ions $\mathrm{RBE}$ at $10 \%$ survival were calculated. Ten of the 11 cell lines were radioresistant with $\mathrm{SF} 2>0.45$. After irradiation with carbon ions, as expected, SF2 were lower (0.08 to 0.38) (11). This study showed that irradiations with photons or carbon ions induced a $G 2 / M$ arrest followed by the formation of multinucleated cells irrespective of the cell line studied, its p53 or O-6-methylguanine-DNA methyltransferase (MGMT) status. The polyploid cells then follow a process of mitotic catastrophe ending in late ceramide dependent apoptotic death (9). The statistical analysis showed that: (I) the photon SF2 and $\mathrm{D}_{10}$ values correlated with those obtained in response to carbon ions; (II) irrespective of cellular radiosensitivity and p53 and MGMT status, release of ceramide is associated with the induction of late apoptosis; and (III) the appearance of polyploid cells after photonic irradiation can predict the RBE of carbon ions (11). This work allowed building up an important database on the cellular and molecular responses of GB cell lines in response to photons and carbon ions. Current experiments focus on DNA lesions followed by $\gamma \mathrm{H} 2 \mathrm{AX}$ immunofluorescence labeling for double strand breaks and alkaline COMET test in order to quantify the single strand breaks and the oxidized bases. All these radiobiological data will be compared to the predictions of the $\mathrm{NanOx}^{\circledR}$ model (41) but also to the predictions of the other models (6), as LEM and MKM already implemented in the clinical TPSs.

\section{Biophysical modelization of cell and tissue responses to particle therapy}

Despite their wide developments, the current approaches still show some shortcomings. Thus, a new theoretical framework, called $\mathrm{NanOx}^{\circledR}$ (41), was created gathering principles from existing approaches, while addressing some of their weaknesses $(42,43)$. $\mathrm{NanOx}^{\circledR}$ is a multiscale model that takes the stochastic nature of radiation at nanometric and micrometric scales fully into account, which has been shown to be drastically important (42-44). It also integrates the chemical aspects of radiation-matter interaction by means of a chemical specific energy, a new quantity determined from the production of reactive chemical species induced by irradiation (45). Such a quantity represents the accumulation of oxidative stress and sublethal damages in the cell, potentially generating non-local lethal events in $\mathrm{NanO}^{\circledR}{ }^{\circledR}$. The complementary local lethal events occur in a very localized region (nanoscale) and, alone, lead to cell death. Both classes of events independently contribute to cell death. The comparison between experimental data and model predictions for the V79 cell line show a good agreement. In particular, the dependence of the typical shoulders of cell survival curves on LET are well described, but also the effectiveness of different ions, including the increase of $\mathrm{RBE}$ and its related overkill effect.

$\mathrm{NanOx}^{\circledR}$ model relies on biological data but also on outputs produced by Monte Carlo simulations that provide data on the deposition of energy $(42,43)$ and the production of radicals (45) at nano and microscopic scales. Moreover, it can provide inputs to models describing the response of tissues or tumors to hadrontherapy. As an example, an estimation of tumor control probability could be derived from the outcomes of LEM model for prostate cancer (46).

\section{Conclusions}

There exists within France HADRON an important activity of applied and translational radiobiology for hadrontherapy, and in particular carbontherapy. The studies carried out are numerous and are developing in different pathways of radiobiology applied to cancer. They range from the more fundamental studies of the mechanisms of radioresistance and tumor relapse, to the production of data intended to constrain the models of calculation of dose and RBE, through frankly translational projects associated with clinical research studies. All these approaches do not have the same 
degree of advancement and maturity as several teams with different histories have come together at different times in their experience in radiobiology. This accretion allowed a variety of approaches which constitute a wealth for France HADRON but also a potential weakness if the projects remain too parallel. The advises of the scientific advisory board of France HADRON are decisive to identify the most important axes and thus allow a convergence of forces on these axes in order to focus the available forces and advance decisively in the resolution of these questions.

\section{Acknowledgements}

Most of the researches reported in the present paper received a contribution either as discussion meetings or direct co-funding by the French research funding agency, Agence Nationale de la Recherche, in the frame of the "Investments for the Future" under the reference: France HADRON, ANR-11-INBS-0007.

\section{Footnote}

Conflicts of Interest: The authors have no conflicts of interest to declare.

\section{References}

1. Bajard M, De Conto JM, Remillieux J, et al. Status of the "ETOILE" project for a French hadrontherapy centre. Radiother Oncol 2004;73 Suppl 2:S211-5.

2. Balosso J, Habrand JL, Montarou G, et al. France HADRON: national infrastructure for hadrontherapy research including ETOILE, ARCHADE and protontherapy centers. Available online: https://hal. archives-ouvertes.fr/hal-01052734/

3. Constanzo J, Fallavier M, Alphonse G, et al. Radiograaff, a proton irradiation facility for radiobiological studies at a 4MV Van de Graaff accelerator. Nucl Instrum Methods Phys Res Sect B Beam Interact Mater At 2014;334:52-8.

4. Dauvergne D, Constanzo J, Fallavier M, et al. Radiograaff: a medium energy proton irradiation platform for radiobiological studies. Presentation and first results. Available online: https://hal.archives-ouvertes.fr/hal01052737/

5. Durantel F, Balanzat E, Cassimi A, et al. Dosimetry for radiobiology experiments at GANIL. Nucl Instrum Methods Phys Res Sect Accel Spectrometers Detect Assoc Equip 2016;816:70-7.
6. Beuve M, Alphonse G, Maalouf M, et al. Radiobiologic parameters and local effect model predictions for headand-neck squamous cell carcinomas exposed to high linear energy transfer ions. Int J Radiat Oncol Biol Phys 2008;71:635-42.

7. Maalouf M, Alphonse G, Colliaux A, et al. Different mechanisms of cell death in radiosensitive and radioresistant p53 mutated head and neck squamous cell carcinoma cell lines exposed to carbon ions and $\mathrm{x}$-rays. Int J Radiat Oncol Biol Phys 2009;74:200-9.

8. Hanot M, Boivin A, Malésys C, et al. Glutathione depletion and carbon ion radiation potentiate clustered DNA lesions, cell death and prevent chromosomal changes in cancer cells progeny. PLoS One 2012;7:e44367.

9. Alphonse G, Maalouf M, Battiston-Montagne P, et al. p53-independent early and late apoptosis is mediated by ceramide after exposure of tumor cells to photon or carbon ion irradiation. BMC Cancer 2013;13:151.

10. Bertrand G, Maalouf M, Boivin A, et al. Targeting head and neck cancer stem cells to overcome resistance to photon and carbon ion radiation. Stem Cell Rev 2014;10:114-26.

11. Ferrandon S, Magné N, Battiston-Montagne P, et al. Cellular and molecular portrait of eleven human glioblastoma cell lines under photon and carbon ion irradiation. Cancer Lett 2015;360:10-6.

12. Hamdi DH, Barbieri S, Chevalier F, et al. In vitro engineering of human $3 \mathrm{D}$ chondrosarcoma: a preclinical model relevant for investigations of radiation quality impact. BMC Cancer 2015;15:579.

13. Laurent C, Leduc A, Pottier I, et al. Dramatic increase in oxidative stress in carbon-irradiated normal human skin fibroblasts. PLoS One 2013;8:e85158.

14. Guy JB, Rancoule C, Méry B, et al. Radiosensitivity and/or radioresistance of head and neck cancers: Biological angle. Bull Cancer 2016;103:41-7.

15. Méry B, Guy JB, Espenel S, et al. Targeting head and neck tumoral stem cells: From biological aspects to therapeutic perspectives. World J Stem Cells 2016;8:13-21.

16. Gilormini M, Wozny AS, Battiston-Montagne P, et al. Isolation and Characterization of a Head and Neck Squamous Cell Carcinoma Subpopulation Having Stem Cell Characteristics. J Vis Exp 2016;(111).

17. Moncharmont C, Levy A, Gilormini M, et al. Targeting a cornerstone of radiation resistance: cancer stem cell. Cancer Lett 2012;322:139-47.

18. Moncharmont C, Levy A, Guy JB, Fet al. Radiationenhanced cell migration/invasion process: a review. Crit 
Rev Oncol Hematol 2014;92:133-42.

19. Moncharmont C, Guy JB, Wozny AS, et al. Carbon ion irradiation withstands cancer stem cells' migration/ invasion process in Head and Neck Squamous Cell Carcinoma (HNSCC). Oncotarget 2016;7:47738-49.

20. Wozny AS, Lauret A, Battiston-Montagne P, et al. Differential pattern of HIF- $1 \alpha$ expression in HNSCC cancer stem cells after carbon ion or photon irradiation: one molecular explanation of the oxygen effect. $\mathrm{Br} \mathrm{J}$ Cancer 2017;116:1340-9.

21. Maucort-Boulch D, Baron MH, Pommier P, et al. Rationale for carbon ion therapy in high-grade glioma based on a review and a meta-analysis of neutron beam trials. Cancer Radiother 2010;14:34-41.

22. Leblond MM, Gérault AN, Corroyer-Dulmont A, et al. Hypoxia induces macrophage polarization and re-education toward an M2 phenotype in U87 and U251 glioblastoma models. Oncoimmunology 2015;5:e1056442.

23. Calipel A, Lux AL, Guérin S, et al. Differential Radiosensitivity of Uveal Melanoma Cell Lines After $\mathrm{X}$-rays or Carbon Ions Radiation. Invest Ophthalmol Vis Sci 2015;56:3085-94.

24. Saintigny Y, Cruet-Hennequart S, Hamdi DH, et al. Impact of therapeutic irradiation on healthy articular cartilage. Radiat Res 2015;183:135-46.

25. Hamdi DH, Chevalier F, Groetz JE, et al. Comparable Senescence Induction in Three-dimensional Human Cartilage Model by Exposure to Therapeutic Doses of X-rays or C-ions. Int J Radiat Oncol Biol Phys 2016;95:139-46.

26. Heinonen M, Guipaud O, Milliat F, et al. Detecting time periods of differential gene expression using Gaussian processes: an application to endothelial cells exposed to radiotherapy dose fraction. Bioinformatics 2015;31:728-35.

27. Yanagi T, Kamada T, Tsuji H, et al. Dose-volume histogram and dose-surface histogram analysis for skin reactions to carbon ion radiotherapy for bone and soft tissue sarcoma. Radiother Oncol 2010;95:60-5.

28. Ferrandon S, Saultier P, Carras J, et al. Telomere profiling: toward glioblastoma personalized medicine. Mol Neurobiol 2013;47:64-76.

29. Chevalier F, Hamdi DH, Saintigny Y, et al. Proteomic overview and perspectives of the radiation-induced bystander effects. Mutat Res Rev Mutat Res 2015;763:280-93.

30. Laprie A, Hu Y, Alapetite C, et al. Paediatric brain tumours: A review of radiotherapy, state of the art and challenges for the future regarding protontherapy and carbontherapy. Cancer Radiother 2015;19:775-89.

31. Pommier P, Balosso J, Lièvre M, et al. EP-1272 Medicoeconomical prospective randomized trials of carbon ions therapy. Radiother Oncol 2012;103:S485.

32. Wozny AS, Alphonse G, Battiston-Montagne P, et al. Influence of Dose Rate on the Cellular Response to Lowand High-LET Radiations. Front Oncol 2016;6:58.

33. Prezado Y, Fois GR. Proton-minibeam radiation therapy: a proof of concept. Med Phys 2013;40:031712.

34. Peucelle C, Nauraye C, Patriarca A, et al. Proton minibeam radiation therapy: Experimental dosimetry evaluation. Med Phys 2015;42:7108-13.

35. Prezado Y, Gonzalez-Infantes W, Heinrich S, et al. SU-GTeP3-09: Proton Minibeam Radiation Therapy Increases Normal Tissue Resistance. Med Phys 2016;43:3675-6.

36. Chargari C, Moncharmont C, Lévy A, et al. Cancer stem cells, cornerstone of radioresistance and perspectives for radiosensitization: glioblastoma as an example. Bull Cancer 2012;99:1153-60.

37. Kobayashi K, Frohlich H, Usami N, et al. Enhancement of X-ray-induced breaks in DNA bound to molecules containing platinum: a possible application to hadrontherapy. Radiat Res 2002;157:32-7.

38. Porcel E, Tillement O, Lux F, et al. Gadolinium-based nanoparticles to improve the hadrontherapy performances. Nanomedicine 2014;10:1601-8.

39. Martínez-Rovira I, Prezado Y. Evaluation of the local dose enhancement in the combination of proton therapy and nanoparticles. Med Phys 2015;42:6703-10.

40. Calugaru V, Nauraye C, Cordelières FP, et al. Involvement of the Artemis protein in the relative biological efficiency observed with the $76-\mathrm{MeV}$ proton beam used at the Institut Curie Proton Therapy Center in Orsay. Int J Radiat Oncol Biol Phys 2014;90:36-43.

41. Cunha M, Monini C, Testa E, et al. NanOx, a new model to predict cell survival in the context of particle therapy. Phys Med Biol 2017;62:1248-68.

42. Beuve M, Colliaux A, Dabli D, et al. Statistical effects of dose deposition in track-structure modelling of radiobiology efficiency. Nucl Instrum Methods Phys Res Sect B Beam Interact Mater At 2009;267:983-8.

43. Beuve M. Formalization and theoretical analysis of the Local Effect Model. Radiat Res 2009;172:394-402.

44. Cunha M, Testa E, Komova OV, et al. Modeling cell response to low doses of photon irradiation--Part 1: on the origin of fluctuations. Radiat Environ Biophys 2016;55:19-30. 
45. Colliaux A, Gervais B, Rodriguez-Lafrasse C, et al. Simulation of ion-induced water radiolysis in different conditions of oxygenation. Nucl Instrum Methods Phys Res Sect B Beam Interact Mater A 2015;365:596-605.

Cite this article as: Rodriguez-Lafrasse C, Saintigny $\mathrm{Y}$, Chevalier F, Bernaudin M, Laurent C, Pouzoulet F, Prezado Y, Megnin-Chanet F, François A, Guipaud O, Beuve M, Balosso J. Translational research in radiobiology in the framework of France HADRON national collaboration. Transl Cancer Res 2017;6(Suppl 5):S795-S806. doi: 10.21037/tcr.2017.06.33
46. Chanrion MA, Sauerwein W, Jelen U, et al. The influence of the local effect model parameters on the prediction of the tumor control probability for prostate cancer. Phys Med Biol 2014;59:3019. 